\title{
THE LEAD SALT QUANTUM DOT INTERMEDIATE BAND SOLAR CELL
}

\author{
Elisa Antolín, Antonio Martí, and Antonio Luque \\ Instituto de Energía Solar, Universidad Politécnica de Madrid, Madrid, Spain
}

\begin{abstract}
We propose a new kind of quantum dot (QD) materials for the implementation of the intermediate band solar cell (IBSC) [1]. The materials are formed by lead salt QDs of the family IV-VI (PbTe, PbSe or PbS) embedded in a semiconductor of the family II-VI $\left(\mathrm{Cd}_{1-x} \mathrm{Mg}_{\mathrm{x}} \mathrm{Te}, \mathrm{Cd}_{\mathrm{x}} \mathrm{Zn}_{1-\mathrm{x}} \mathrm{Te}\right.$, and $\mathrm{CdS}_{1-\mathrm{x}} \mathrm{Se}_{\mathrm{x}}$ or $\mathrm{ZnSe}_{1-\mathrm{x}} \mathrm{Te}_{\mathrm{x}}$, respectively). These QDs are not nucleated due to lattice mismatch, as it is the case of the $\ln A s / G a A s$ QD material system grown by the Stranski-Krastanov (S-K) mode. In these materials, the QDs precipitate due to the difference in lattice type: the QD lead salt material crystallizes in the rocksalt structure, while the II-VI host material has the zincblende structure [2]. Therefore, it is possible to use lattice-matched QD/host combinations, avoiding all the strain-related problems found in previous QD-IBSC developments. In this paper we discuss the properties of the lead salt QD materials and propose that they are appropriate to overcome the fundamental drawbacks of present III-Vbased QD-IBSC prototypes. We also calculate the band diagram for some examples of IV-VI/II-VI QD materials. The detailed balance efficiency limit of QD-IBSCs based on the studied materials is found to be over $60 \%$ under maximum concentration.
\end{abstract}

\section{INTRODUCTION}

The reasons why quantum dot (QD) materials were proposed ten years ago [3] to implement the intermediate band solar cell (IBSC) [1] are still valid today. To achieve a high efficiency, the IBSC requires a material that: (i) enables sub-bandgap photon absorption, to increase the photocurrent with respect to a conventional solar cell, and (ii) does not suffer from excessive non radiative recombination, to preserve a similar output voltage (see Figure 1 and the explanation in its caption). QDs are in principle suitable for both things: (i) the inter-band and intra-band optical transitions between QD confined levels are (relatively) strong and allow the absorption of subbandgap light of any polarization, and (ii) when coherent (defect-free) islands are grown, QDs have a radiative behavior (they do not act as Shockley-Read-Hall recombination sites as atom impurities do, because they do not have the same vibration modes).

Although the theoretical scheme has not changed, we know now more about the practical operation of QD-IBSC prototypes and the properties of QD materials that are critical for that operation. During the last years several groups have worked on the development of the QD-IBSC using InAs/GaAs-based materials [4-10]. These QD materials have allowed demonstrating the physical principles of IBSC operation at low temperatures [5, 6]. Also, in some cases it has been possible to measure an increase in the photocurrent with respect to reference samples without QDs [7, 8, 10]. But there are fundamental limitations that prevent the InAs/GaAs-based QD-IBSC from achieving a really high efficiency $[9,11]$.

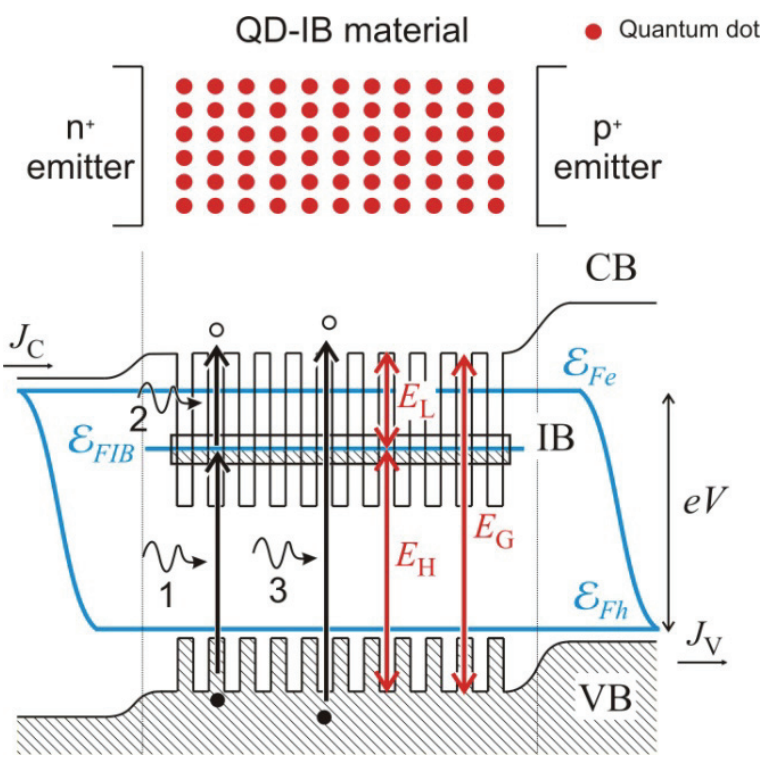

Figure 1 Simplified band diagram of an IBSC illustrating its operation. The intermediate band (IB) divides the semiconductor gap y two sub-bandgaps ( $E_{\mathrm{L}}$ and $E_{\mathrm{H}}$ ) and enables the absorption of extra photons (labeled 1 and 2). The emitters are required to pin the electron and hole quasi-Fermi levels $\left(\varepsilon_{\mathrm{Fe}}\right.$ and $\left.\varepsilon_{\mathrm{Fh}}\right)$ at the contacts to preserve the voltage. For this to occur, the quasi-Fermi level of IB carriers $\left(\varepsilon_{\mathrm{FIB}}\right)$ has to be split from the other two under operation, which implies a sufficient recombination lifetime in all three transitions ( $C B \rightarrow V B, C B \rightarrow I B$ and $I B \rightarrow V B$ ).

The gap of GaAs, $1.4 \mathrm{eV}$, is too low to fabricate a very efficient IBSC. A gap of about $2 \mathrm{eV}$ would be required. But that is not the only limiting factor. Most of the problems of the InAs/GaAs-based IBSC are strain-related. The QD synthesis is based on the Stranski-Krastanov (S-K) growth mode, in which nano-islands nucleate on top of a thin wetting layer (WL) at a certain critical thickness [12]. This process is triggered by the elastic relaxation of the strain that appears between the two materials, which have a large lattice mismatch. However, as depicted in Figure 2, the band diagram is severely affected by the presence of strain. The InAs gap is blue-shifted from 0.36 to $\sim 0.7 \mathrm{eV}$, 
and the sub-gaps become extremely asymmetric: $E_{H}$ is typically over $1 \mathrm{eV}$, while $E_{\mathrm{L}}$ is in the range of $0.2 \mathrm{eV}$. This makes that the electron populations of $\mathrm{CB}$ and IB (i.e. $\varepsilon_{\mathrm{Fe}}$ and $\left.\varepsilon_{\mathrm{FIB}}\right)$ cannot be split at room temperature. The situation is worsened because the strain also induces a degradation of the shape of the dots, which tend to have a very wide base and small height. This shape results in a high number of extra confined states for electrons. We have identified that this situation reduces the absorption in intraband $(I B \rightarrow C B)$ transitions [13], and enhances the thermal connection between IB and CB [11, 14]. Besides, the existence of a WL also degrades the QD material properties because it contributes to a fast non-radiative relaxation of carriers from the CB to the IB [14].

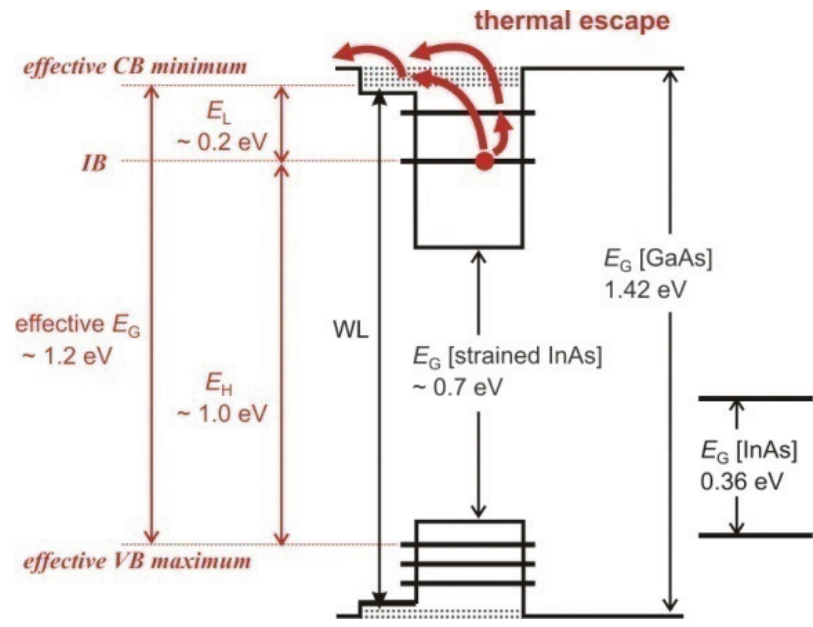

Figure 2 Simplified InAs/GaAs QD band diagram including the effects of strain and the WL. On the left are shown the corresponding effective bandgaps of an IBSC fabricated with that QD material system. On the right is included the band diagram of bulk (unstrained) InAs for comparison.

The fact that the quasi-Fermi levels $\varepsilon_{\mathrm{Fe}}$ and $\varepsilon_{\mathrm{FIB}}$ cannot be split at room temperature is a fundamental drawback of present QD-IBSC prototypes. It implies that the voltage preservation predicted by the IBSC theoretical model cannot be realized. It has been demonstrated that practical InAs/GaAs QD-IBSCs can achieve voltage preservation at low temperatures [6].

With these considerations in mind, there are two possible strategies for the development of the QD-IBSC. One is to continue using the III-V S-K approach, but introducing important variations in the QD material system; for example, by adding $\mathrm{N}$ to the dot and substituting the $\mathrm{GaAs}$ host by a wider gap material (GalnP, AlGaAs) as proposed in [15]. To maintain the S-K approach means most likely to keep fighting the effects of strain with techniques as reported in [8, 9, 16, 17]. The second strategy is to move away from the InAs/GaAs QD family towards a completely different set of materials. This work proposes the lead salt/II-VI QD material family as a candidate for the second strategy.

\section{THE LEAD SALT QD MATERIAL SYSTEMS}

The lead salt QD materials are synthesized by a completely different technique than the S-K method. In this case the QD and host materials have closely matched lattice parameters. Both have the same face-centered cubic symmetry, but the bonding configuration is different: lead salts crystallize in the sixfold coordinated rocksalt lattice and the host materials in the fourfold coordinated zincblende lattice. This lattice-type mismatch results in a large miscibility gap, i.e. for a range of synthesis temperatures, lead salt QDs precipitate in the host zincblende material.

The most studied IV-VI/II-VI QD material system is $\mathrm{PbTe} / \mathrm{CdTe}$. The QDs are typically synthesized in a twostep method: first, a PbTe/CdTe multilayer stack is epitaxially grown, and second, the material is annealed to make the QDs precipitate [2]. In this approach the size of the dots is determined by the thickness of the PbTe layers grown. Diameters as small as $5 \mathrm{~nm}$ and areal densities over $10^{11} \mathrm{~cm}^{-2}$ can be achieved from a $1 \mathrm{~nm}$ PbTe epitaxial layer [18]. Figure 3 illustrates how a QD-IBSC would be produced using this method. Recently, it has been also demonstrated that similar $\mathrm{PbTe} / \mathrm{CdTe} \mathrm{QDs}$ can be synthesized by $\mathrm{Pb}^{+}$-implantation in $\mathrm{CdTe}$ and subsequent annealing [19], and by self-organization in-situ during epitaxial growth at the appropriated temperatures [20].

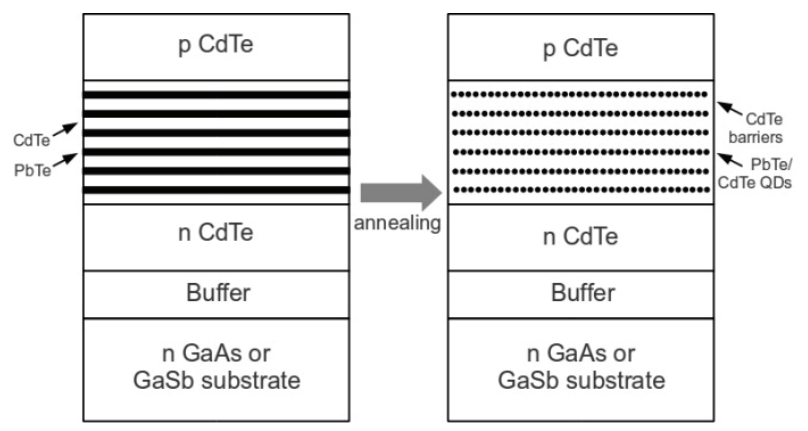

Figure 3 Example of a lead salt QD-IBSC structure using $\mathrm{PbTe} / \mathrm{CdTe} \mathrm{QDs}$ as IB material, and CdTe p- and n-emitters.

It has been demonstrated that $\mathrm{PbTe} / \mathrm{CdTe} \mathrm{QDs}$ are WLfree and can have highly centrosymmetric shapes (almost spherical) [2], they have atomically sharp interfaces [21] and they are strongly luminescent up to over 400K [20-22]. As the QD and host materials are almost lattice-matched (see Figure 4) a large number of layers separated by thin spacers can be grown.

Currently the $\mathrm{PbTe} / \mathrm{CdTe} \mathrm{QD}$ material is being investigated for the fabrication of infrared emitters. In that application it is being exploited the inter-band radiative 
transitions (from the confined electron ground-state to the confined hole ground-state). The photon energy of that emission is typically about $0.4 \mathrm{eV}$ [22], while the inter-band emission of InAs/GaAs QDs is the range of $1 \mathrm{eV}$ (see Figure 2). As it can be seen in Figure 4, the bulk gaps of InAs/GaAs and $\mathrm{PbTe} / \mathrm{CdTe}$ are practically the same, and the huge difference in emission wavelength is explained by the presence of strain in the III-V system and the absence of it in the lead salt system.

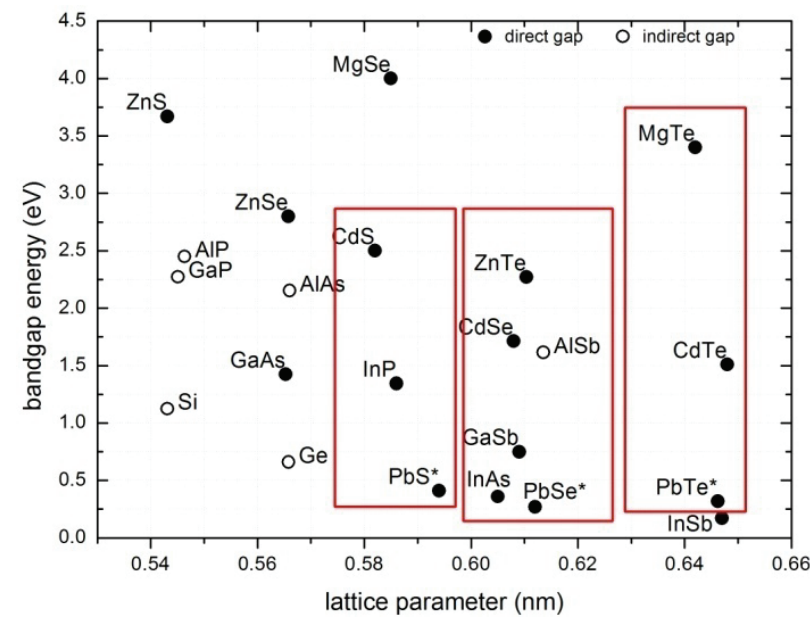

Figure 4 Bandgap vs. lattice parameter map, including most used binary compounds of the III-V, II-VI and VIVI families, and the diamond-like semiconductors SiGe. All values correspond to the zincblende structure, except those marked with *, which correspond to the rocksalt crystal structure.

The three red boxes in the $E_{\mathrm{G}}$ vs. lattice constant map of Figure 4 mark three possible families for implementation of a IV-VI/II-VI QD-IBSC:

(i) PbTe QDs can be embedded in a $\mathrm{Cd}_{1-\mathrm{x}} \mathrm{Mg}_{\mathrm{x}} \mathrm{Te}$ alloy. The reason to add $\mathrm{Mg}$ to the host material is twofold. On the one hand, it increases the host gap, making it approach 2 $\mathrm{eV}$, which increases the detailed balance efficiency limit of the IBSC. On the other hand, it improves the latticematching between QD and host (in PbTe/CdTe there is a small mismatch of $0.3 \%$ ). This is expected to improve the material quality if a thick QD stack is to be grown.

(ii) A second possibility is to embed PbSe dots in a CdSe or ZnTe matrix. To improve the bandgap distribution and lattice matching, again a small amount of $\mathrm{Mg}$ could be added to the host or an optimized $\mathrm{Cd}_{1-x} \mathrm{Zn}_{\mathrm{x}} \mathrm{Te}_{\mathrm{y}} \mathrm{Se}_{1-\mathrm{y}}$ alloy could be attempted.

(iii) Finally, PbS QDs could be embedded in a $\mathrm{CdS}_{1-\mathrm{x}} \mathrm{Se}_{\mathrm{x}}$ or $\mathrm{ZnSe}_{1-\mathrm{x}} \mathrm{Te}_{\mathrm{x}}$ host.

To our knowledge, combinations of the types (ii) and (iii) using $\mathrm{PbSe}$ and $\mathrm{PbS}$ have not been synthesized yet. The self-organization of $\mathrm{PbSe}$ and $\mathrm{PbS}$ islands by lattice- mismatch epitaxy on III-V substrates has already been demonstrated [23, 24]. A reason to choose one of these two families would be to avoid the use of Te, which is scarce. Also, they can have the advantages over the PbTe approach of minimizing or even eliminating the use of $\mathrm{Cd}$, and also, of a higher matching to common substrates, such as GaSb, GaAs and $\mathrm{Si}$ (although it is well-known that CdTe can be also epitaxially grown over GaSb or GaAs with the highest quality by inserting a suitable buffer layer). On the other hand, compared to the already explored $\mathrm{PbTe} / \mathrm{CdTe} \mathrm{QD}$ system, they present an uncertainty with respect to the synthesis. It is known that $\mathrm{PbSe}$ is immiscible in CdSe in a similar way than $\mathrm{PbTe}$ in $\mathrm{CdTe}$ [25]. But for other combinations, it will be necessary to perform studies on the solubilities and formation thermodynamics in order to evaluate which stoichiometries and conditions lead to a favorable precipitation of lead salt QDs.

We can summarize the following advantages of the IV$\mathrm{VI} / \mathrm{II}-\mathrm{VI}$ QD materials for the implementation of the IBSC:

(a) If the QD and host materials are close to latticematching, there is no significant strain accumulation. Therefore, the crystalline quality can be very high in the QD stack and in the emitter grown on top of it. Nonradiative recombining defects are minimized and the number of QD layers - and subsequently, the subbandgap absorption strength - can be enhanced with respect to the $\operatorname{InAs} / \mathrm{GaAs}$ approach.

(b) In the II-VI group there are direct-gap materials with much larger gap than in the III-V group (see Figure 4). $\mathrm{ZnTe}$, for example, is a widely used II-VI compound with $E_{G}=2.27 \mathrm{eV}$. In order to have an IBSC with optimized bandgap distribution and really high efficiency limit it is required a host material with large fundamental gap.

(c) There is no wetting layer.

(d) The lack of strain between the QD and the host eliminates the problems illustrated in Figure 2 for the InAs/GaAs QD system. The QD material gap is not blueshifted (as a matter of fact, that would not be the case even under lattice mismatch, because lead salts have an anomalous behavior by which their gap decreases as pressure is applied [26]). Another consequence of the lack of strain is the possibility to grow small, centrosymmetric QDs (aspect ratio 1) [2, 20-22]. On the other hand, lead salts have small effective masses for both electron and holes, and therefore, the number of confined levels is small for both kinds of carriers (they are direct gap semiconductors with almost symmetrical bands at the $L$ point minimum). All these factors together enable an advantageous band diagram for IBSC implementation: $E_{\mathrm{H}}$ and $E_{\mathrm{L}}$ can be maximized, while the number of undesired extra confined states can be minimized. This will be illustrated with some example calculations in the next section. 
Basing on the argumentations given in the previous section, it is expected that the band diagram of IV-VI QDS helps to preserve the voltage in QD-IBSCs at higher temperatures than it is possible with the InAs/GaAs approach, and eventually, that it enables the attainment of high conversion efficiencies at room temperature.

\section{BAND DIAGRAM CALCULATIONS}

We have chosen two simple examples of IV-VI QD materials to evaluate their potential for implementation of a QD-IBSC: the fully lattice-matched $\mathrm{PbTe} / \mathrm{Cd}_{1-\mathrm{x}} \mathrm{Mg}_{\mathrm{x}} \mathrm{Te}$ combination and $\mathrm{PbSe} / \mathrm{ZnTe}$. For both systems, the QD confined levels have been calculated under the effective mass approximation. As the QDs are highly centrosymmetric they can be approximated by a spherical geometry [22]. We have neglected strain effects because, as discussed before, their impact on the band diagram is not significant for the evaluation we are performing.

Table 1 Material parameters used for the $\mathrm{PbTe} /$ $\mathrm{Cd}_{1-\mathrm{x}} \mathrm{Mg}_{\mathrm{x}} \mathrm{Te} \mathrm{QD}-\mathrm{IBSC}(\mathrm{T}=300 \mathrm{~K})$ and references.

\begin{tabular}{|l|c|c|}
\cline { 2 - 3 } \multicolumn{1}{c|}{} & PbTe & $\mathrm{Cd}_{1-\mathrm{x}} \mathrm{Mg}_{\mathrm{x}} \mathrm{Te}$ \\
\hline \hline$E_{\mathrm{G}}(\mathrm{eV})$ & $0.32[22]$ & $1.51+1.35 x+0.54 x^{2}[27]$ \\
\hline$a(\mathrm{~nm})$ & $0.6462[28]$ & $0.648-0.006 x[27]$ \\
\hline$m_{\mathrm{e}}{ }^{*} / m_{0}$ & $m_{t}=0.024$ & $\frac{1}{m_{l}^{\Gamma}(x)}=\frac{x}{0.17}+\frac{1-x}{0.09}[27]$ \\
& $m_{l}=.24[29]$ & $\frac{1}{m_{\mathrm{HH}}(x)}=\frac{x}{0.80}+\frac{1-x}{0.82}[27]$ \\
\hline$m_{\mathrm{h}}{ }^{*} / m_{0}$ & $m_{t}=0.022$ & $5.78-0.67 x[30,31]$ \\
\hline$\Phi(\mathrm{eV})$ & \multicolumn{2}{|c|}{0.135 (between PbTe and CdTe) [28] } \\
\hline$\Delta E_{\mathrm{V}}(\mathrm{eV})$ & \multicolumn{2}{|c|}{$0.129]$} \\
\hline
\end{tabular}

Table 2 Material parameters used for the $\mathrm{PbSe} /$ ZnTe QD-IBSC ( $\mathrm{T}=300 \mathrm{~K})$ and references.

\begin{tabular}{|l|c|c|}
\cline { 2 - 3 } \multicolumn{1}{c|}{} & $\mathrm{PbSe}$ & ZnTe \\
\hline \hline$E_{\mathrm{G}}(\mathrm{eV})$ & $0.27[32]$ & $2.27[27]$ \\
\hline$a(\mathrm{~nm})$ & $0.612[33]$ & $0.61037[27]$ \\
\hline$m_{\mathrm{e}}{ }^{*} / m_{0}$ & $\begin{array}{c}m_{t}=0.04 \\
m_{l}=0.07[29]\end{array}$ & $0.117[27]$ \\
\hline$m_{\mathrm{h}}{ }^{*} / m_{0}$ & $\begin{array}{c}m_{t}=0.034 \\
m_{l}=.068[29]\end{array}$ & $0.67[27]$ \\
\hline$\Phi(\mathrm{eV})$ & \multicolumn{2}{|c|}{0.12 (between PbSe and PbTe) [26] } \\
\hline$\Delta E_{\mathrm{V}}(\mathrm{eV})$ & \multicolumn{2}{|c}{} \\
\hline
\end{tabular}

The material parameters used in the calculations are compiled in Table 1 and Table 2. For calculating the effective masses of the rock-salt materials, we have used the spherical average between the transversal $\left(m_{\mathrm{t}}\right)$ and longitudinal $\left(m_{1}\right)$ masses [22]: $m^{*}=m_{\mathrm{t}}^{2 / 3} m_{\mathrm{l}}^{1 / 3}$. In the case of zinc-blend materials we have only regarded the heavy hole effective mass $\left(m_{\mathrm{HH}}{ }^{*}\right)$.

In this kind of calculation, the heterojunction band offsets $\left(\Delta E_{\mathrm{C}}\right.$ and $\left.\Delta E_{\mathrm{V}}\right)$ are parameters of main importance, but they are often not clearly established in the literature. Given the relative lack of information in the case of lead salts, for compiling tables 1 and 2 we have used the following set of data: (1) the energy between VB and vacuum level $(\Phi)$ for the host materials (linearly interpolated in the case of $\mathrm{Cd}_{1-\mathrm{x}} \mathrm{Mg}_{\mathrm{x}} \mathrm{Te}$ ); (2) a reported VB offset for a given material combination. In the first example, to achieve full lattice matching with the PbTe QD material $\mathrm{Mg}$ content of $x=0.3$ is required in the $\mathrm{Cd}_{1}$ ${ }_{x} \mathrm{Mg}_{\mathrm{x}} \mathrm{Te}$ matrix. The resulting $\mathrm{QD} /$ host band alignment is shown in Figure 5.

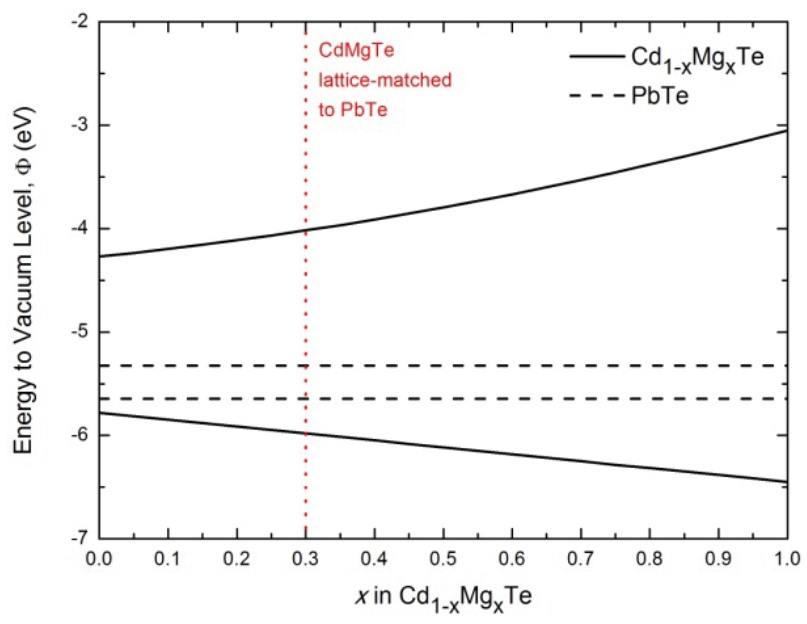

Figure 5 Calculated room temperature energies of the CB and VB of $\mathrm{Cd}_{1-\mathrm{x}} \mathrm{Mg}_{\mathrm{x}} \mathrm{Te}$ as a function of the $\mathrm{Mg}$ content $x$ (solid line). The energy of PbTe bands is included to show the heterojunction band alignment (dashed lines).

QD levels for the lattice-matched $\mathrm{PbTe} / \mathrm{Cd}_{0.7} \mathrm{Mg}_{0.3}$ Te system

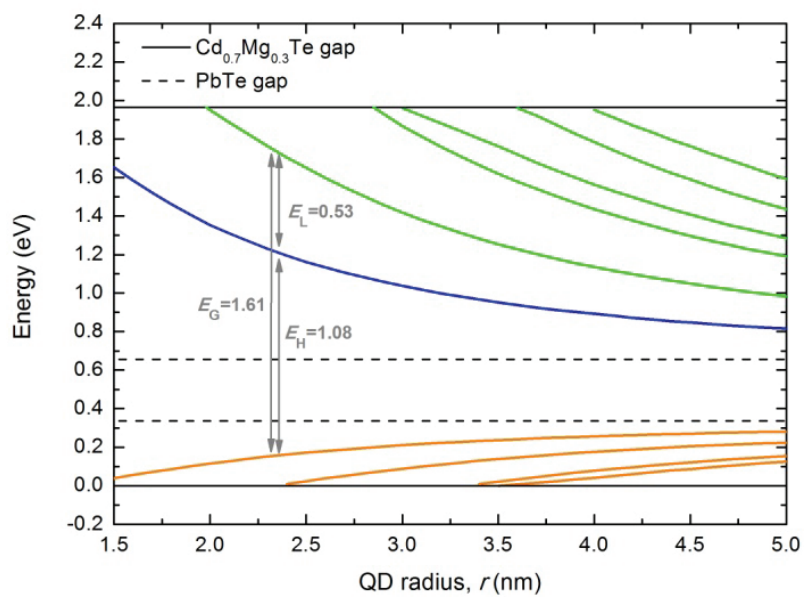

Figure 6 Calculated energy levels as a function of the QD radius for the $\mathrm{PbTe} / \mathrm{Cd}_{0.7} \mathrm{Mg}_{0.3} \mathrm{Te} \mathrm{QD}$ system. The CdMgTe stoichiometry has been chosen to achieve exact lattice-matching to $\mathrm{PbTe}$ (red dotted line on

Figure 5). The gaps labeled $\left(E_{\mathrm{G}}, E_{\mathrm{H}}, E_{\mathrm{L}}\right)$ correspond to the optimum case $(r=2.3 \mathrm{~nm})$ according to detailed balance calculations. 
Figure 6 and Figure 7 show the calculated QD confined states in dependence with the QD radius, $r$, for the $\mathrm{PbTe} / \mathrm{Cd}_{0.7} \mathrm{Mg}_{0.3} \mathrm{Te}$ and the $\mathrm{PbSe} / \mathrm{ZnTe}$ systems, respectively. In a QD-IBSC implementation the fundamental electron levels $\left(E^{\mathrm{e}}{ }_{10}\right)$ serve as IB. In our analysis we consider that the excited electron levels $\left(E^{e}{ }_{n}\right.$, $n>1)$ and the hole levels $\left(E_{n l}^{h}\right)$ are not thermally isolated from the respective $C B$ or VB edges. Therefore, the first electron exited state $E_{20}^{e}$ (hole ground state $E_{10}^{h}$ ) is considered the effective CB (VB) band edge, except for very small dots where those levels do not appear. For both the $\mathrm{PbTe}$ and $\mathrm{PbSe}$ cases, we find appropriated energy band diagrams for IBSC fabrication if $r$ is small. As previously anticipated, the reasons are the large gap of the host material and the narrow gap of the QD material (not altered by strain) together with the small effective masses.

$\mathrm{QD}$ levels for the $\mathrm{PbSe} / \mathrm{ZnTe}$ system



Figure 7 Calculated energy levels as a function of the $\mathrm{QD}$ radius for the in the $\mathrm{PbSe} / \mathrm{ZnTe} \mathrm{QD}$ system. The gaps labeled $\left(E_{\mathrm{H}}, E_{\mathrm{L}}\right)$ correspond to the optimum case $(r=1.7 \mathrm{~nm})$ according to detailed balance calculations.

\section{EFFICIENCY LIMIT CALCULATIONS}

The band diagrams obtained in the previous section have been used to calculate the theoretical efficiency limit of a QD-IBSC fabricated with the two example materials. We have used the ideal detailed balance model of the IBSC expounded in [1], assuming maximum concentration $(46050 \mathrm{X})$ and full absorption selectivity among the three optical transitions. The solar spectrum is approximated by the $6000 \mathrm{~K}$ black-body spectrum. As it is known, under these conditions the absolute efficiency limit of the IBSC (optimized gap distribution) is $63.2 \%$, to be compared with the Shockley-Queisser limit of $40.7 \%$ for a single-gap cell.

Table 3 shows the maximum detailed balance efficiencies calculated with the band diagrams of Figure 6 and Figure 7. They correspond to an optimized dot radius, which in both cases is $\sim 2 \mathrm{~nm}$. The resulting gap values are also marked on the figures. In both cases the efficiency limit is $>60 \%$. Therefore, it can be concluded that the IV-VI/II-VI QD materials are promising for QD-IBSC implementation also from the point of view of detailed balance efficiency limit.

Table 3 Detailed balance efficiency limit (n) under maximum concentration calculated for two IV-VI/II-VI QD-IBSCs and optimized parameters leading to that efficiency limit.

\begin{tabular}{|c|c|c|c|}
\hline & $\begin{array}{c}\text { QD radius } \\
(\mathrm{nm})\end{array}$ & Gaps (eV) & $\eta(\%)$ \\
\hline $\mathrm{PbTe} / \mathrm{Cd}_{0.7} \mathrm{Mg}_{0.3} \mathrm{Te}$ & 2.3 & $\begin{array}{l}E_{G}=1.61 \\
E_{H}=1.08 \\
E_{L}=0.53\end{array}$ & 60.7 \\
\hline $\mathrm{PbSe} / \mathrm{ZnTe}$ & 1.7 & $\begin{array}{l}E_{\mathrm{G}}=2.26 \\
E_{\mathrm{H}}=1.45 \\
E_{\mathrm{L}}=0.81\end{array}$ & 60.2 \\
\hline
\end{tabular}

\section{CONCLUSIONS}

A new family of materials has been proposed for the implementation of the intermediate band solar cell: the lattice-matched, but lattice-type-mismatched, IV-VI/II-VI QD materials. With these materials, a strain-free wide-gap QD-IBSC can be fabricated, and therefore, they have the potential to overcome the most relevant limitations of current InAs/GaAs-based QD-IBSC prototypes. The confined QD levels of two example QD material systems, $\mathrm{PbTe} / \mathrm{Cd}_{0.7} \mathrm{Mg}_{0.3} \mathrm{Te}$ and $\mathrm{PbSe} / \mathrm{ZnTe}$, have been calculated. Suitable band diagrams have been obtained for IBSC implementation, especially for small QDs (radius $\sim 2 \mathrm{~nm}$ ). The detailed balance efficiency limit under maximum concentration calculated for a QD-IBSC based on any of the two materials is higher than $60 \%$.

\section{ACKNOWLEDGEMENTS}

This work has been supported by the project IBPOWER funded by the European Commission (Grant No. 211640), by the project NANOGEFES funded by the Spanish Ministerio de Ciencia e Innovación (ENE2009-14481-C0201 ) and by the project NUMANCIA-2 funded by the Regional Government of Madrid (S2009/ENE1477).

\section{REFERENCES}

[1] A. Luque and A. Martí, "Increasing the efficiency of ideal solar cells by photon induced transitions at intermediate levels", Phys. Rev. Lett. 78, pp. 5014-5017, 1997.

[2] W. Heiss, H. Groiss, E. Kaufmann, et al., "Centrosymmetric $\mathrm{PbTe} / \mathrm{CdTe}$ quantum dots coherently embedded by epitaxial precipitation", Appl. Phys. Lett. 88, p. 192109, 2006.

[3] A. Martí, L. Cuadra, and A. Luque, "Quantum dot intermediate band solar cell", in Conference Record of the 
28th IEEE Photovoltaics Specialists Conference, New York, USA, 2000, pp. 940-943.

[4] A. Luque, A. Martí, N. López, et al., "Experimental analysis of the quasi-Fermi level split in quantum dot intermediate-band solar cells", Appl. Phys. Lett. 87, pp. 083505-3, 2005.

[5] A. Martí, E. Antolín, C. R. Stanley, et al., "Production of Photocurrent due to Intermediate-to-Conduction-Band Transitions: A Demonstration of a Key Operating Principle of the Intermediate-Band Solar Cell", Phys. Rev. Lett. 97, p. 247701, 2006.

[6] E. Antolín, A. Martí, P. G. Linares, et al., "Advances in quantum dot intermediate band solar cells", in Photovoltaic Specialists Conference (PVSC), 2010 35th IEEE, 2010, pp. 65-70.

[7] S. A. Blokhin, A. V. Sakharov, A. M. Nadtochy, et al., "AlGaAs/GaAs photovoltaic cells with InGaAs quantum dot arrays", Physics and Semiconductor Technique 43, pp. 537-542, 2009.

[8] R. Oshima, A. Takata, and Y. Okada, "Straincompensated InAs/GaNAs quantum dots for use in highefficiency solar cells", Appl. Phys. Lett. 93, p. 083111, 2008.

[9] V. Popescu, G. Bester, M. C. Hanna, et al., "Theoretical and experimental examination of the intermediate-band concept for strain-balanced (In, Ga)As/Ga(As,P) quantum dot solar cells", Phys. Rev. B 78, p. 205321, 2008.

[10] S. M. Hubbard, C. G. Bailey, C. D. Cress, et al., "Short circuit current enhancement of GaAs solar cells using strain compensated InAs quantum dots ", in Conference record of the 33rd IEEE Photovoltaic Specialists Conference, San Diego, USA, 2008.

[11] A. Luque, A. Martí, E. Antolín, et al., "Radiative thermal escape in intermediate band quantum dot solar cells", AIP Advances 1, p. 0225125, 2011.

[12] D. Leonard and M. Krishnamurthy, "Direct formation of quantum-sized dots from uniform coherent islands of InGaAs on GaAs surfaces", Appl. Phys. Lett. 63, p. 3203, 1993.

[13] A. Luque, A. Martí, E. Antolín, and P. García-Linares, "Intraband Absorption for Normal Illumination in Quantum Dot Intermediate Band Solar Cells", Solar Energy Materials and Solar Cells 94, pp. 2032-2035, 2010.

[14] E. Antolín, A. Martí, C. D. Farmer, et al., "Reducing carrier escape in the InAs/GaAs quantum dot intermediate band solar cell", Journal of Applied Physics 108, p. 064513, Sep 2010.

[15] P. G. Linares, A. Martí, E. Antolín, and A. Luque, "III-V compound semiconductor screening for implementing quantum dot intermediate band solar cells", Journal of Applied Physics 109, p. 014313, 2011.

[16] R. B. Laghumavarapu, M. El-Emawy, N. Nuntawong, et al., "Improved device performance of InAs/GaAs quantum dot solar cells with strain compensation layers", Appl. Phys. Lett. 91, p. 243115, 2007.

[17] S. M. Hubbard, C. D. Cress, C. G. Bailey, et al., "Effect of strain compensation on quantum dot enhanced GaAs solar cells", Appl. Phys. Lett. 92, p. 123512, Mar 2008.
[18] H. Groiss, E. Kaufmann, G. Springholz, et al., "Size control and midinfrared emission of epitaxial $\mathrm{PbTe} / \mathrm{CdTe}$ quantum dot precipitates grown by molecular beam epitaxy", Appl. Phys. Lett. 91, p. 222106, 2007.

[19] E. Kaufmann, T. Schwarzl, H. Groiss, et al., "PbTe and SnTe quantum dot precipitates in a CdTe matrix fabricated by ion implantation", J. Appl. Phys. 106, p. 043105, 2009.

[20] K. Koike, H. Harada, T. Itakura, et al., "Photoluminscence characterization of $\mathrm{PbTe} / \mathrm{CdTe}$ quantum dots grown by lattice-type mismatched epitaxy", Journal of Crystal Growth 301-302, pp. 722-725, 2007.

[21] W. Heiss, H. Groiss, E. Kaufmann, et al., "Quantum dots with coherent interfaces between rocksalt-PbTe and zincblende-CdTe", J. Appl. Phys. 101, p. 081723, 2007.

[22] T. Schwarzl, E. Kaufmann, G. Springholz, et al., "Temperature-dependent midinfrared photoluminescence of epitaxial $\mathrm{PbTe} / \mathrm{CdTe}$ quantum dots and calculation of the corresponding transition energy", Phys. Rev. B 78, pp. $165320-9,2008$.

[23] A. B. Preobrajenski, K. Barucki, Chass, et al., "Exploiting the Difference in Lattice Structures for Formation of Self-Assembled PbS Dots on $\operatorname{lnP}(110) "$, Physical Review Letters 85, p. 4337, 2000.

[24] X. L. Huang, Z. Labadi, A. Hammiche, and A. Krier, "Growth of self-assembled PbSe quantum-dots on GaSb(100) by liquid phase epitaxy", J. Phys. D: Appl. Phys. 35, pp. 3091-3095, 2002.

[25] Z. F. Tomashik, G. S. Oleinik, and V. N. Tomashik, "The phase diagram of the PbSe-CdSe system", Inorganic Materials 16, pp. 168-170, 1980.

[26] S.-H. Wei and A. Zunger, "Electronic and structural anomalies in lead chalcogenides", Physical Review B 55, p. $13605,1997$.

[27] S. Adachi, Properties of Semiconductor Alloys Group IV, III-V, II-VI Semiconductors: Wiley, 2009.

[28] J. Si, S. Jin, H. Zhang, et al., "Experimental determination of valence band offset at $\mathrm{PbTe} / \mathrm{CdTe}(111)$ heterojunction interface by $x$-ray photoelectron spectroscopy", Appl. Phys. Lett. 93, p. 202101, 2008.

[29] G. Martinez, M. Schlüter, and M. L. Cohen, "Electronic structure of $\mathrm{PbSe}$ and $\mathrm{PbTe}$. I. Band structures, densities of states, and effective masses", Physical Review B 11, p. 651, 1975.

[30] R. K. Swank, "Surface Properties of II-VI Compounds", Physical Review 153, p. 844, 1967.

[31] S.-H. Wei and A. Zunger, "Calculated natural band offsets of all II-VI and III-V semiconductors: Chemical trends and the role of cation $d$ orbitals", Appl. Phys. Lett. 72, pp. 2011-2013, 1998.

[32] J. N. Zemel, J. D. Jensen, and R. B. Schoolar, "Electrical and Optical Properties of Epitaxial Films of PbS, $\mathrm{PbSe}, \mathrm{PbTe}$, and SnTe", Physical Review 140, p. A330, 1965.

[33] G. A. Grinbom, M. Saraf, C. Saguy, et al., "Density of states in a single $\mathrm{PbSe} / \mathrm{PbS}$ core-shell quantum dot measured by scanning tunneling spectroscopy", Physical Review B 81, p. 245301. 